\title{
DMTB: A comprehensive online resource of 16S rRNA genes, ecological metadata, oligonucleotides, and magnetic properties of magnetotactic bacteria
}

\author{
LIN Wei ${ }^{1,2}$, LI Bi $^{1,2} \&$ PAN YongXin ${ }^{1,2}$ \\ ${ }^{1}$ Paleomagnetism and Geochronology Laboratory, Key Laboratory of the Earth's Deep Interior, Institute of Geology and Geophysics, Chinese \\ Academy of Sciences, Beijing 100029, China; \\ ${ }^{2}$ France-China Bio-Mineralization and Nano-Structures Laboratory, Institute of Geology and Geophysics, Chinese Academy of Sciences, Beijing \\ 100029, China
}

\begin{abstract}
Magnetotactic bacteria (MTB) are of interest in microbiology, biomineralization, advanced magnetic materials, and bio-geosciences because of their ability to form highly ordered intracellular magnetic minerals. Great strides for MTB studies have been made in the past four decades. In this paper we complied the first internet-accessible database for MTB, Database of Magnestotactic Bacteria (DMTB). It contains information of 16S rRNA gene sequences, corresponding ecological metadata, oligonucleotides, and magnetic properties of MTB. The comprehensive information contained in DMTB will provide a very useful data resource for researchers from different disciplines. The website of DMTB is at http://database.biomnsl.com/.
\end{abstract}

magnetotactic bacteria, biomineralization, database

Citation: Lin W, Li B, Pan Y X. DMTB: A comprehensive online resource of 16S rRNA genes, ecological metadata, oligonucleotides, and magnetic properties of magnetotactic bacteria. Chinese Sci Bull, 2011, 56: 476-478, doi: 10.1007/s11434-010-4275-0

Magnetotactic bacteria (MTB) can sense the Earth's magnetic field and navigate the oxic-anoxic transition zone in chemically stratified environments through intracellular organelles termed magnetosomes [1]. Magnetosomes are ferrimagnetic nano-sized iron minerals, magnetite $\left(\mathrm{Fe}_{3} \mathrm{O}_{4}\right)$ or greigite $\left(\mathrm{Fe}_{3} \mathrm{~S}_{4}\right)$, that are surrounded by a lipid bilayer membrane, and are arranged into one or more chains. Because of their distinctive genetic control on the synthesis of magnetosomes, MTB have served as a model for understanding biologically controlled mineralization processes [1]. The superior crystalline structure and uniform grain size $(35-120 \mathrm{~nm})$ make magnetosomes potentially novel biomaterials for a number of applications. Moreover, given their widespread distribution in fresh, brackish and marine waters, and their production of iron minerals, MTB may play significant roles in geological iron and sulfur cycling, as well as in bulk magnetism of sediments [2].

In the past decades, research efforts from various fields have greatly advanced our understanding of MTB, especially with regard to tractable genetic systems and genome analyses of several pure cultures, morphological and molecular

*Corresponding author (email: yxpan@mail.iggcas.ac.cn) diversity and ecology of uncultivated MTB, and magnetic properties of MTB cells (for a recent review see [3]). Based on phylogenetic results, all currently known MTB are classified within the Proteobacteria and Nitrospirae phyla. More than two hundred 16S rRNA gene sequences of MTB have been reported from more than 30 sampling locations, and 20 probes have been designed for identification of MTB through fluorescence in situ hybridization (FISH). Because magnetic approaches are fast, effective, and nondestructive in distinguishing bacterial crystals from abiogenic magnetic minerals in different environments, many magnetic methods have been applied. Currently, more than 600 articles concerning MTB have been published in peer-reviewed journals. The rapidly-growing data on MTB are stimulating broadening interdisciplinary studies in such fields as global biogeography, comparative genomics, evolutionary analysis, and paleoenvironmental research. However, to our knowledge, no database specifically focused on MTB has yet been established. The great amount of data in different publications and several general databases (e.g. GenBank) make it difficult and time-consuming to screen and compile data for multi-disciplinary studies. Thus, a comprehensive and easily accessible database of MTB is needed for multi-disciplinary 
scientists.

Through the present study, we developed the Database of Magnetotactic Bacteria (DMTB) containing published 16S rRNA gene sequences, ecological metadata, oligonucleotides (PCR primers and FISH probes), and magnetic properties of MTB. This database provides easy and fast access to comprehensive information on MTB.

For compiling 16S rRNA gene sequences, a complex combination of keywords [(SSU OR 16S OR small subunit) AND (rRNA OR rDNA OR ribosomal RNA OR rRNA gene) AND (magnetic bacterium OR magnetotactic bacterium OR magneto*)] was used to search available sequences in GenBank database. The retrieved sequences were then examined and selected based on their definitions. A total of 264 sequences were selected for the DMTB (up to the end of October, 2009). Seven sequences from the scientific literature, which were missing from the above search strategy, were manually added to the DMTB. Among all selected 271 sequences, 23 were defined as uncultivated MTB sequences by submitters; however, our analysis shows low sequence identities $(<75 \%)$ with known MTB sequences that were from pure cultures or non-cultured with confirmation by FISH. We marked these sequences as 'Potential MTB' in the DMTB, and their origins need to be confirmed by further studies. All data for ecological metadata of sampling sites, oligonucleotides, and magnetic properties were collected from the scientific literature.

Data catalogs of the DMTB are summarized in Figure 1. By the initial release of the DMTB (November, 2009), the database contained 271 16S rRNA gene sequences, environmental information on 29 sampling sites, 3 sets of specific primers, $20 \mathrm{FISH}$ probes, and 10 entries of magnetic information. The data are accessible through four main interfaces: Site Sort, Phylo Sort, Oligonucleotides (PCR primers and FISH probes), and Magnetic Properties.

Because of the rapidly increasing accumulation of sequence information and ecological metadata, environmental microbiology has become increasingly more important in this area of research, especially studies on the biogeography of microorganisms [4,5]. In the database, therefore, we provided information of site- and phylogeny-based arrangements of 16S rRNA genes of MTB.

Site Sort interface contains a complete list of sampling sites in alphabetical order, listing the country and the number of sequences. The entry of each site name is linked to an individual page containing detailed information about environmental metadata. Each number of sequence entries consists of information on 16S rRNA gene sequences of MTB. Sequence names also are linked to individual FASTA files for each 16S rRNA gene sequence. GenBank accession numbers and references are directed to the corresponding GenBank description interface and PubMed abstracts, respectively.

Phylo Sort interface lists taxonomic phyla and classes with which MTB are affiliated. Each entry includes a list of MTB sequence information, which is classified using the Greengenes "classify" utility. Identified MTB belong to six classes, including Alphaproteobacteria (153 sequences), Betaproteobacteria (2 sequences), Gammaproteobacteria (20 sequences), Deltaproteobacteria (65 sequences), Epsilonproteobacteria (3 sequences), and Nitrospirales (5 sequences). Sequence names, GenBank accession numbers, and references in each entry serve as links to specific pages within public databases.

Oligonucleotides interface includes FISH probes and PCR primers specific for MTB. Each entry consists of the oligonucleotide name, target position, sequence, length, degeneracy, $\mathrm{G}+\mathrm{C}$ content, and basic usage information for probes or primers. The corresponding reference is linked to the PubMed database.

Magnetic Properties interface contains magnetic information. Each MTB sample entry contains a variety of relevant information, including the Verwey transition temperature $\left(T_{\mathrm{v}}\right)$, delta ratio $\left(\delta_{\mathrm{FC}} / \delta_{\mathrm{ZFC}}\right)$, saturation magnetization $\left(M_{\mathrm{s}}\right)$,

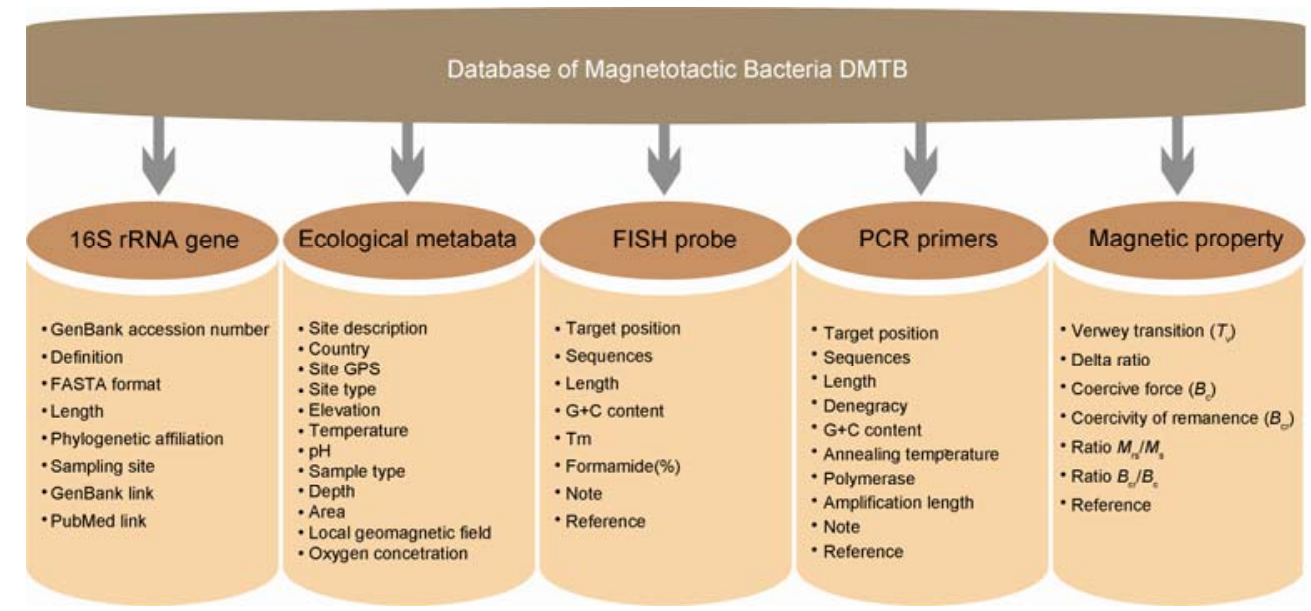

Figure 1 The database structure of DMTB. FISH, fluorescence in situ hybridization; $M_{\mathrm{s}}$, saturation magnetization; $M_{\mathrm{rs}}$, saturation remanence; $B_{\mathrm{c}}$, coercive force; and $B_{\mathrm{cr}}$, coercivity of remanence. 
saturation remanence $\left(M_{\mathrm{rs}}\right)$, coercive force $\left(B_{\mathrm{c}}\right)$, coercivity of remanence $\left(B_{\mathrm{cr}}\right)$, ratios of $M_{\mathrm{rs}} / M_{\mathrm{s}}$ and $B_{\mathrm{cr}} / B_{\mathrm{c}}$, and related references.

Search queries of the entire DMTB can be initiated easily with various keywords, such as site name, sequence name, accession number, and the last name of the first author.

In summary, the new DMTB offers an online accessible database, including comprehensive information on $16 \mathrm{~S}$ rRNA gene sequences, ecological metadata, oligonucleotides, and magnetic properties of MTB. This database will be a valuable resource for microbiologists, ecologists, biomineralogists, biogeophysicists, and physicists for further study the biogeography, diversity, community structure, evolution, mineralogy, and physical characteristics of MTB.
The DMTB can be accessed freely through the Web at http://database.biomnsl.com/.

We sincerely thank Prof. Long-Fei Wu at CNRS-Marseille, France, for his valuable comments. This work was supported by the Knowledge Innovation Program of the Chinese Academy of Sciences (80912910), the National Natural Science Foundation of China (40821091), and the CAS/SAFEA International Partnership Program for Creative Research Teams (KZCX2-YW-T10).

1 Faivre D, Schüler D. Chem Rev, 2008, 108: 4875-4898

2 Pan Y, Deng C, Liu Q, et al. Chinese Sci Bull, 2004, 49: 2563-2568

3 Schüler D. Magnetoreception and Magnetosomes in Bacteria. New York: Springer Verlag, 2007

4 Martiny J B H, Bohannan B J M, Brown J H, et al. Nat Rev Microbiol, 2006, 4: 102-112

5 Green J L, Bohannan B J M, Whitaker R J. Science, 2008, 320: $1039-1043$

Open Access This article is distributed under the terms of the Creative commons Attribution License which permits any use, distribution, and reproduction in any medium, provided the original author(s) and source are credited. 